\title{
Axigluon signatures at hadron colliders
}

\author{
Germán Rodrigo * \\ Instituto de Física Corpuscular, CSIC-Universitat de València, \\ Apartado de Correos 22085, E-46071 Valencia, Spain. \\ E-mail: german.rodrigodific.uv.es
}

\begin{abstract}
Axigluons are colored heavy neutral gauge boson that are predicted by some theories. The most important model-independent manifestation of axigluons is the generation of a forward-backward asymmetry in top-antitop quark production at $p \bar{p}$ collisions which originates from the charge asymmetry. We update our previous analysis for the inclusive QCD induced forward-backward asymmetry and define a new observable which is more sensitive to the effect than the forwardbackward asymmetry. We find a lower limit of $1.4 \mathrm{TeV}$ at $90 \%$ C.L. on the axigluon mass from recent measurements of the asymmetry at Tevatron, and extend the analysis to LHC in suitably selected samples. Like at Tevatron, the charge asymmetry can probe larger values of the axigluon mass than the dijet mass distribution.
\end{abstract}

8th International Symposium on Radiative Corrections

October 1-5, 2007

Florence, Italy

* Supported by Consejo Superior de Investigaciones Científicas (CSIC) under grant PIE 200650I247, Ministerio de Educación y Ciencia (MEC) under grant FPA2007-60323 and CSD2007-00042, Generalitat Valenciana under grant GVACOMP2007-156, and European Commission MRTN FLAVIAnet under contract MRTN-CT-2006-035482. 


\section{Introduction}

The Large Hadron Collider (LHC) will enter into operation very soon, allowing to explore the existence of new physics at the TeV energy scale with unprecedented huge statistics [1]. Since the top quark is the heaviest known elementary particle it plays a fundamental role in many extensions of the Standard Model (SM), and its production and decay channels are promising probes of new physics. The total cross section of top-antitop quark production at LHC is about 100 times larger than at Tevatron. This will lead to the production of millions of $t \bar{t}$ pairs per year even at the initial low luminosity of $\mathscr{L}=10^{33} \mathrm{~cm}^{-2} \mathrm{~s}^{-1}$ (equivalent to $10 \mathrm{fb}^{-1} /$ year integrated luminosity).

Some properties of the top quark can be studied at Tevatron through the forward-backward asymmetry which originates from the charge asymmetry $[2,3]$. The Born processes relevant for top quark production, $q \bar{q} \rightarrow t \bar{t}$ and $g g \rightarrow t \bar{t}$, do not discriminate between final quark and antiquark, thus predicting identical differential distributions also for the hadronic production process. At order $\alpha_{s}^{3}$ however a charge asymmetry is generated and the differential distributions of top quarks and antiquarks are no longer equal. A similar effect leads also to a strange-antistrange quark asymmetry, $s(x) \neq \bar{s}(x)$, through next-to-next-to-leading (NNLO) evolution of parton densities [4]. The inclusive charge asymmetry has its origin in two different reactions: radiative corrections to quark-antiquark annihilation (Fig. 1) and interference between different amplitudes contributing to gluon-quark scattering $q g \rightarrow t \bar{t} q$ and $\bar{q} g \rightarrow t \bar{t} \bar{q}$. Gluon-gluon fusion remains obviously symmetric. The integrated forward-backward asymmetry has been predicted to be about $+5 \%$ at Tevatron [2]; with the top quarks emitted preferentially in the direction of the incoming protons. This prediction suffers, however, from a sizable uncertainty because, although arising from a one-loop calculation and the corresponding real emission terms, it is still a leading order (LO) result.

At LHC the total forward-backward asymmetry vanishes trivially because the proton-proton initial state is symmetric. A charge asymmetry is, however, still visible in suitably defined distributions [2].

The forward-backward asymmetry of top quarks has already been measured at Tevatron $[5,6$, 7, 8]. The latest CDF analysis [6] based on $1.7 \mathrm{fb}^{-1}$ integrated luminosity, which supersedes the results presented in Ref. [7], gives for the inclusive asymmetry

$$
A\left(\Delta y \cdot Q_{l}\right)=0.28 \pm 0.13 \text { (stat) } \pm 0.05 \text { (sys) },
$$

where the charge asymmetry is defined by the difference in the number of events with positive and negative $\Delta y \cdot Q_{l}$, the rapidity difference of the semileptonically and hadronically decaying top quark times the charge of the charged lepton. The inclusive asymmetry, although compatible with the theoretical prediction, is still statistically dominated. The statistical error, is expected to be reduced to 0.04 with $8 \mathrm{fb}^{-1}$ [5], which is comparable with the systematic error.

The measurement at D0 [8] with $0.9 \mathrm{fb}^{-1}$ integrated luminosity gives for the uncorrected asymmetry

$$
A_{\mathrm{FB}}^{\mathrm{obs}}=0.12 \pm 0.08 \text { (stat) } \pm 0.01 \text { (sys) } .
$$

Like CDF, this analysis use $y_{t}-y_{\bar{t}}$ as sensitive variable. In Ref. [8] upper limits on $t \bar{t}+X$ production via a $Z^{\prime}$ resonance are also provided. Measurements of the exclusive asymmetry of the four- and five-jet samples are also given in both analysis. 
Models which extend the standard color gauge group to $S U(3)_{L} \times S U(3)_{R}$ at high energies, the so called chiral color theories [9], predict the existence of a massive, color-octet gauge boson, the axigluon, which couples to quarks with an axial vector structure and the same strong interaction coupling strength as QCD. Although there are many different implementations of chiral color theories with new particles in varying representations of the gauge groups, the most important model-independent prediction of these models is the existence of the axigluon, where its main signature is the appearance of a charge asymmetry of order $\alpha_{s}^{2}$. Because the coupling of the axigluon to quarks is an axial vector coupling the charge asymmetry that can be generated is maximal.

\section{The QCD induced charge asymmetry}

The QCD induced charge asymmetry in the reaction $q \bar{q} \rightarrow t \bar{t}(g)$ is generated by the interference of final-state with initial-state gluon radiation [Fig. 1, (a) $\times(\mathrm{b})$ ] and by the interference of virtual box diagrams with the Born process [Fig. 1, (c) $\times(\mathrm{d})$ ]. The virtual plus soft radiation on one hand and the real hard radiation on the other contribute with opposite signs, with the former always larger that the latter such that the inclusive asymmetry becomes positive. Top quarks are thus preferentially emitted in the direction of the incoming quark at the partonic level, which translates to a preference in the direction of the incoming proton in $p \bar{p}$ collisions. Flavour excitation $g q(\bar{q}) \rightarrow t \bar{t} X$ generates already at tree-level a forward-backward asymmetry which at Tevatron is also positive although one order of magnitude smaller than the asymmetry from $q \bar{q}$ annihilation.

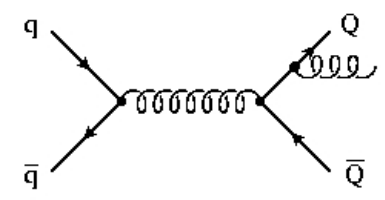

(a)

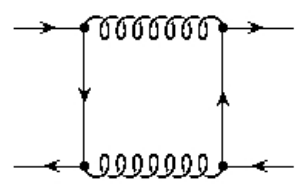

(c)

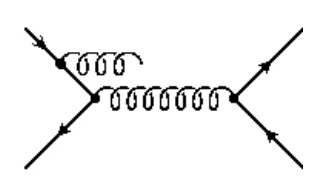

(b)

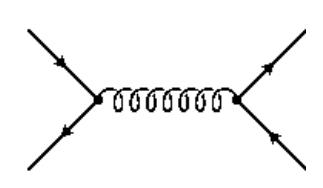

(d)

Figure 1: Origin of the QCD charge asymmetry in hadroproduction of heavy quarks: interference of finalstate (a) with initial-state (b) gluon bremsstrahlung, plus interference of the double virtual gluon exchange (c) with the Born diagram (d). Only representative diagrams are shown.

Updating our previous analysis [2], we predict for the total charge asymmetry at $\sqrt{s}=1.96 \mathrm{TeV}$ :

$$
A=\frac{N_{t}(y \geq 0)-N_{\bar{t}}(y \geq 0)}{N_{t}(y \geq 0)+N_{\bar{t}}(y \geq 0)}=0.051(6) .
$$

We have also proposed [10] a new differential distribution, the pair asymmetry, that leads to an enhancement of the charge asymmetry. Selecting events where the rapidities $y_{+}$and $y_{-}$of both the 

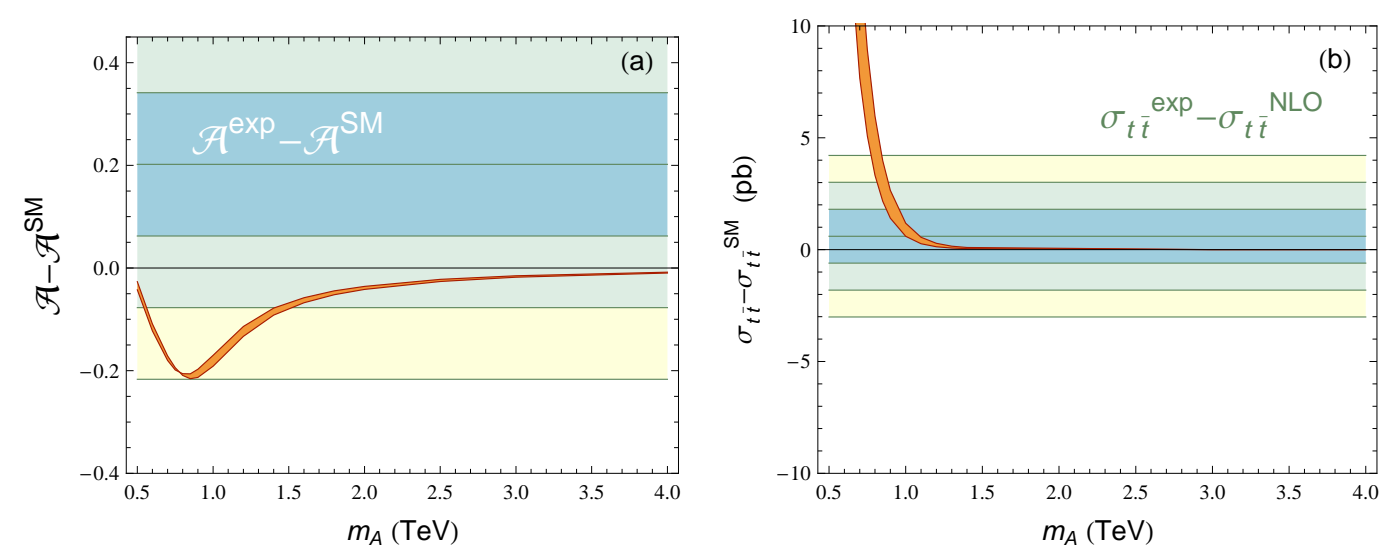

Figure 2: Comparison of the axigluon contribution to the top quark pair asymmetry (a), and to the topantitop total cross section (b) with the $1 \sigma, 2 \sigma$ and $3 \sigma$ contours as a function of the axigluon mass.

top and antitop quarks have been determined, we define $Y=1 / 2\left(y_{+}+y_{-}\right)$as average rapidity, and consider the differential pair asymmetry $\mathscr{A}(Y)$ for all events with fixed $Y$ as a function of $Y$. For the integrated pair asymmetry we predict [10]:

$$
\mathscr{A}=\frac{\int d Y\left(N_{\mathrm{ev} .}\left(y_{+}>y_{-}\right)-N_{\mathrm{ev} .}\left(y_{+}<y_{-}\right)\right)}{\int d Y\left(N_{\mathrm{ev} .}\left(y_{+}>y_{-}\right)+N_{\mathrm{ev} .}\left(y_{+}<y_{-}\right)\right)}=0.078(9) .
$$

The integrated pair asymmetry is equivalent to the definition of the asymmetry used in Refs. [6, 8]. The reason for the enhancement of the effect can be understood as follows: by defining the pair asymmetry one essentially investigates the forward-backward asymmetry in the $t \bar{t}$ rest frame, where the forward-backward asymmetry amounts to $7-8.5 \%$ [2], depending on $\hat{s}$. This value is largely recovered by considering the pair asymmetry $\mathscr{A}(Y)$, independently of $Y$. In contrast, events where both $t$ and $\bar{t}$ are produced with positive and negative rapidities do not contribute to the integrated forward-backward asymmetry $A$, which is therefore reduced to around $5 \%$.

\section{Axigluon limits from Tevatron}

The interference between the gluon and axigluon induced amplitudes for the reaction $q \bar{q} \rightarrow t \bar{t}$ does not contribute to the production cross section. However, it generates a charge asymmetry that gives rise to a forward-backward asymmetry in $p \bar{p}$ collisions in the laboratory frame [11]. The square of the axigluon amplitude is symmetric and contributes to the total cross-section, which will show a typical resonance peak in the top-antitop invariant mass distribution [12]. While the interference term is suppressed by the squared axigluon mass $1 / m_{A}^{2}$, the contribution of the square of the axigluon amplitude will be suppressed by $1 / m_{A}^{4}$. It is therefore obvious that the forwardbackward asymmetry is potentially sensitive to larger values of the axigluon mass than the topantitop dijet distribution. Gluon-gluon fusion is not affected by the axigluon exchange because there are no direct gluon-axigluon vertices with an odd number of axigluons [12] due to parity. 

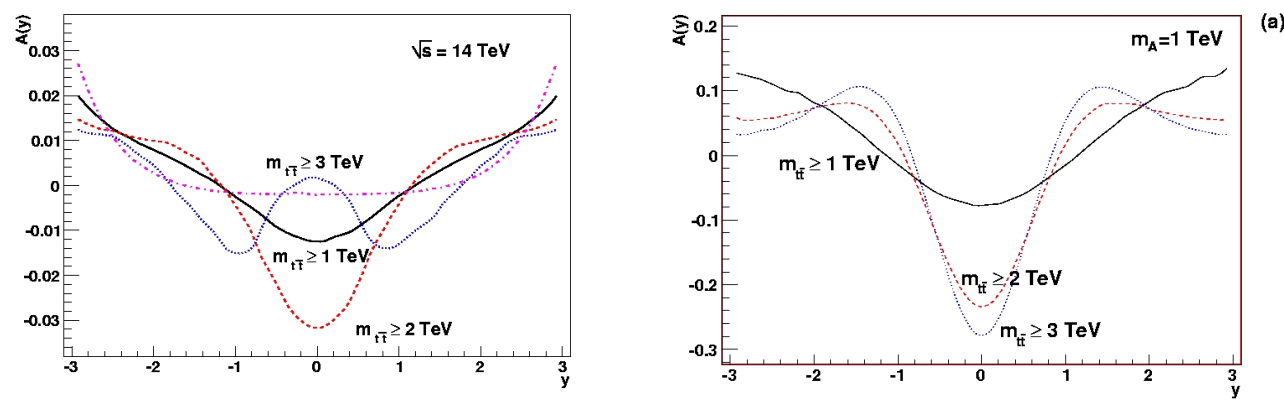

Figure 3: Differential top quark charge asymmetry at LHC generated by QCD (left) and by the exchange of a massive axigluon (right) for top-antitop quark invariant masses larger than $2 m_{t}$ (dotted-dashed), $1 \mathrm{TeV}$ (solid), $2 \mathrm{TeV}$ (dashed), and $3 \mathrm{TeV}$ (dotted). Factorization and renormalization scales set to $\mu=m_{t}$.

Figs. 2(a) and 2(b) show the contribution of axigluon exchange in comparison to the experimental measurement of the integrated pair asymmetry (Eq. (1.1)), and of the top-antitop total cross section [13], $\sigma_{t \bar{t}}=7.3 \pm 0.9(\mathrm{pb})$, respectively. For the SM prediction we use Eq. (2.2), and $\sigma_{t \bar{t}}^{\mathrm{NLO}}=6.7 \pm 0.8(\mathrm{pb})[14]$.

We observe that above $m_{A}>1 \mathrm{TeV}$ the contribution of the axigluon to the total cross section is almost suppressed. Better determinations of the total cross section will not lead to a significant improvement in the bound of the axigluon mass. On the contrary, the pair asymmetry is particularly sensitive to axigluon masses below 2-2.5 TeV, and little improvements can lead to a significant change in the lower bound. We can establish a lower bound of $m_{A}>1.4 \mathrm{TeV}$ at $90 \%$ C.L.

\section{QCD and axigluon induced asymmetries at the LHC}

Top quark production at LHC is forward-backward symmetric in the laboratory frame as a consequence of the symmetric colliding proton-proton initial state. Furthermore, the total cross section is dominated by gluon-gluon fusion and thus the charge asymmetry generated from the $q \bar{q}$ and $g q(g \bar{q})$ reactions is negligible in most of the kinematic phase-space. The effect can be studied nevertheless by selecting appropriately chosen kinematic regions. We have therefore analyzed the effect of selecting samples with high invariant masses of the top-antitop quark pair. Those samples should have a higher amount of $q \bar{q}$ induced events, and an enhanced axigluon contribution even for large $m_{A}$. Thus a sizable asymmetry is expected, although at the price of reducing the total event rate. This should not be a problem at LHC due to the huge top-antitop quark yields.

Fig. 3 illustrates the QCD and the axigluon contributions to the differential charge asymmetry as a function of the top quark rapidity. We have defined a new charge asymmetry where only the the central region is taken into account:

$$
A_{C}\left(y_{C}\right)=\frac{\sigma_{t}\left(|y| \leq y_{C}\right)-\sigma_{\bar{t}}\left(|y| \leq y_{C}\right)}{\sigma_{t}\left(|y| \leq y_{C}\right)+\sigma_{\bar{t}}\left(|y| \leq y_{C}\right)} .
$$

Notice that $A_{C}\left(y_{C}\right)$ vanishes if the whole rapidity spectrum is integrated. A maximum is reached at about $y_{C}=1$. Predictions for different kinematical cuts and axigluon masses can be found in Ref. [10]. 


\section{Summary}

We have updated our previous analysis of the forward-backward and the charge asymmetry in top quark production at hadron colliders. We have also proposed a new observable, the pair asymmetry, where the effect at the Tevatron is enhanced by about a factor 1.5. We have shown that the pair asymmetry is more sensitive to larger axigluon masses that the total top-antitop quark cross section. Preliminary results on the charge asymmetry from the Tevatron lead to a limit on the axigluon mass of $1.4 \mathrm{TeV}$ at $90 \%$ C.L. The analysis has been extended to the LHC. Restricting the event sample to regions of large $t \bar{t}$ mass large axigluon masses can be explored.

\section{Acknowledgements}

I want to thank J.H. Kühn and O. Antuñano for a fruitful collaboration, and the local organizing Committee of RADCOR07 and the Galileo Galilei Institute for Theoretical Physics for their kind hospitality.

\section{References}

[1] F. Gianotti and M. L. Mangano, arXiv:hep-ph/0504221.

[2] J. H. Kühn and G. Rodrigo, Phys. Rev. D 59, 054017 (1999); Phys. Rev. Lett. 81, 49 (1998).

[3] F. Halzen, P. Hoyer and C. S. Kim, Phys. Lett. B 195 (1987) 74.

[4] S. Catani, D. de Florian, G. Rodrigo and W. Vogelsang, Phys. Rev. Lett. 93 (2004) 152003.

[5] T. A. Schwarz, Ph.D. Thesis, University of Michigan, FERMILAB-THESIS-2006-51, UMI-32-38081.

[6] D. Hirschbühl, T. Müller, T. Peiffer, J. Wagner-Kuhr, W. Wagner, and J. Weinelt [CDF Collaboration], CDF Note 8963. R. Erbacher [CDF Collaboration], at International Symposium On Lepton-Photon Interactions at High Energy (LP07), 13-18 Aug 2007, Daegu, Korea.

[7] J. Weinelt, Masters thesis, Universität Karlsruhe, FERMILAB-MASTERS-2006-05; IEKP-KA-2006-21.

[8] V. M. Abazov et al. [D0 Collaboration], arXiv:0712.0851 [hep-ex]. A. Harel [D0 Collaboration], at International Europhysics Conference on High Energy Physics (EPS-HEP2007), Manchester, England, 19-25 Jul 2007; D0 Note 5393-CONF.

[9] J. C. Pati and A. Salam, Phys. Lett. B 58 (1975) 333. L. J. Hall and A. E. Nelson, Phys. Lett. B 153 (1985) 430. P. H. Frampton and S. L. Glashow, Phys. Lett. B 190 (1987) 157; Phys. Rev. Lett. 58 (1987) 2168.

[10] O. Antuñano, J. H. Kuhn and G. V. Rodrigo, Phys. Rev. D 77 (2008) 014003.

[11] L. M. Sehgal and M. Wanninger, Phys. Lett. B 200 (1988) 211.

[12] J. Bagger, C. Schmidt and S. King, Phys. Rev. D 37 (1988) 1188.

[13] S. Cabrera [CDF Collaboration], FERMILAB-CONF-06-356-E.

[14] M. Cacciari, S. Frixione, M. L. Mangano, P. Nason and G. Ridolfi, JHEP 0404 (2004) 068. 